\title{
On Direct Comparing of Medical Guidelines with Electronic Health Record ${ }^{\star}$
}

\author{
Jana Zvárová, Arnošt Veselý, Petr Hanzliček, Josef Špidlen, and \\ David Buchtela
}

European Center for Medical Informatics, Statistics and Epidemiology, Institute of Computer Science AS CR, Pod Vodárenskou věží 2, Praha 8, The Czech Republic

zvarova@euromise.cz

\begin{abstract}
Electronic Health Record (EHR) systems are now being developed in many places. The EuroMISE Center has developed a system of the Multimedia Distributed Electronic Health Record (MUDR) that provides universal structure for collecting data and multimedia objects. Moreover, a method to compare data items on the input of EHR system with medical guidelines has been developed. Therefore it is possible to reveal if the input data item, that represents patient diagnosis or proposed patient treatment, contradicts with medical guidelines or not. The method on direct comparing of medical guidelines with EHR has been tested in hospital information system settings and in settings of the remote GP office.
\end{abstract}

\section{Introduction}

The motivation to use electronic data storage in medicine is based on the obvious drawback of the paper medical record including illegible handwriting, poor organization of documents, missing data and ambiguous data. Current developments in electronic health records focus on advanced features, including strategies for data entry and retrieval, multiple views and transparent integration of diverse information sources of different platforms [1], 2]. Moreover, the availability of an EHR (Electronic Health Record) is a must for using advanced decision support in medicine and health care, including medical guideline support. The European project I4C of the $4^{\text {th }}$ Framework Programme (1996-1998) was carried out for the further advancement of cardiac care [3]. It focused on clinical applications and its main goals were to integrate access to data wherever stored, to support evidence-based care by remote electronic consultation and peer review and to record more comprehensively and more consistently patient data, images, videos and bio-signals. With the support of the $\mathrm{I} 4 \mathrm{C}$ project the multimedia Open Record for Care (ORCA) was developed. It integrates structured patient data entry including history, medication, symptoms and more with multimedia objects as ECG, angiography or laboratory data.

\footnotetext{
* The research was partially supported with the project LN00B107 Ministry of Education CR.
} 
Based on the experience gathered in the EU projects, especially TripleC [4] and MGT [5], 6] the EuroMISE Centre has been focused on specific tasks of EHR, Electronic Formalized Medical Guidelines (EFMG), data mining, decision support and knowledge based systems and on the practical application of new developments in the Czech healthcare environment. There is running development of EHR named MUDR (MUltimedia Distributed electronic health Record), see e.g. 7], 88. The universal architecture of MUDR EHR provides a flexible platform for storage of medical data of any type and MUDR EHR takes into account obligatory standards for Czech health care providers. Moreover, since the EHR serves as a tool for data collection, decision support programs can use its data for automatic generation of alerts, reminders and suggestions when standards of care (e.g. based on medical guidelines) are not achieved. The development of EFMG and the task on making them available for physicians using Internet, especially with the simultaneous use of EHR is desirable.

\section{MUDR Architecture}

The MUDR EHR is based on a three-layer architecture with a data layer, an application layer and a user interface. This decomposition enables separating different system modules to small functional parts, which makes the system more flexible. In our system we define the global architecture with communication interfaces based predominately on XML and HTTP. We also define an interface for connecting medical guidelines formalized in the form of dynamic libraries. These libraries extend the capabilities of the MUDR EHR.

MUDR $^{\text {II }}$ architecture extends the basic three-layer architecture. The communication between the MUDR DB Server and the MUDR Application Layer Service uses the MUDR DB Connection Module, which enables implementing the data layer on various database platforms. The application layer chooses the right module for the database currently used. Using this scheme, the application layer communicates transparently with the data layer using always the identical interface.

The main difference lies in the application layer interface, used to provide the functionality of the application layer. The application layer integrates communication modules to communicate with different client types. The main communication module is called MUDR WS. This module provides objects using the MUDR .NET Remoting API (MUDRNRAPI). Using .NET Remoting, the remote call of methods of the shared objects is possible. This functionality is utilized by another application layer component called MUDR Web Service. This web service makes the MUDR Web Service Application Interface (MUDRWSAPI) accessible to common clients. A typical general practitioner (GP) uses a $\mathrm{PC}$ on his table to work with the MUDR EHR remotely. The communication is enabled by the HTTP Server installed on the application server. The commands and parameters are encoded using the SOAP standard.

For the eventual usage of MUDR thin clients in the form of HTML and WAP browsers we use a MUDR WS Proxy Service. This service is implemented as a 
Common Gateway Interface (CGI) program; it provides classical HTML or WAP pages to thin clients and appears in the role of a classical MUDR Web Service client at the other side.

\section{$3 \quad$ Electronic Formalized Medical Guidelines}

Many medical guidelines were elaborated to improve quality of medical care and to achieve standardization of treatment. Usually, the first version of medical guidelines is worked out by a group of medical experts in a free text. For computer implementation and processing it is necessary to have medical guidelines explicitly structured and developed in an electronic form. A lot of modeling means were suggested for this purpose. The most important and nowadays mostly used is the GLIF (Guideline Interchange Format) model. The GLIF model is a result of collaboration among Columbia University, Harvard University, McGill University and Stanford University. Version 2.0 of GLIF (GLIF2) was published in 1998 [9].

The main goal of GLIF was to enable sharing of electronic formalized medical guidelines (EFMG) among health institutions and across computer applications. GLIF specifies an object-oriented model for guidelines representation and syntax for guidelines utilization in software systems as well as for their transport. GLIF guidelines are mostly given as a flowchart representing a temporarily ordered sequence of steps. Different types of steps in the flowchart represent clinical actions and decisions [9], [10]. To enhance usability of GLIF, new version GLIF3 was worked out. GLIF3 builds upon the framework set by GLIF2, but augments it by introducing several new constructs and extending GLIF2 constructs to allow a more formal definition of decision criteria and patient data [11]. The GLIF model gives process-oriented view of guidelines. The model consists of a set of classes for guideline entities, attributes of these classes and data types for attribute values. Instances of classes (objects) have only attributes and no methods.

A particular guidelines encoded in GLIF are an instance of a general guideline model. It could be represented in a form of oriented graph. The nodes of the graph are guideline steps and edges represents continuation from one step to the other one. Guideline steps are action step, decision step, branch and synchronization steps and patient state step. Action steps specify clinical actions that are to be performed. It can be an application of some therapy, carrying out some examination or measurement etc. Action step also may name subguidelines, which provide greater detail for the action. Decision steps are used for conditional branching. There are two kinds of decision steps: case step and choice step. Case step is used, when branching is determined by evaluation of defined logical criteria based on data items. Choice step is used when the decision cannot be precisely specified in guidelines themselves and decision should be made by the user. Branch and synchronization steps enable concurrency in the model. Guideline steps that follow branch step could be performed concurrently. Branches with root in branch step eventually converge in synchronization step. 
In this step all branches are synchronized. It means, that actions, that follow the synchronization step, could not be performed, unless all actions, following branch step and preceding the synchronization step, are finished. Patient state step characterizes patient's clinical state.

Guideline model can be equivalently expressed also in a language form. Syntax for guideline describing language is a part of guideline model specification. In language form encoded guidelines consist of a sequence of guideline steps. Some attributes of a guideline step contain next guideline steps. It enables sequential representation of graph structure in the guideline describing language.

Action steps of medical guidelines specify different kinds of physician actions:

- An action could mean to carry out some examination or some laboratory measurement. The gained results we will call medical guideline examination data.

- An action could promote some treatment or application of some therapy. Such actions we will call medical guideline actions.

Patient state steps of medical guidelines specify patient states, for example diagnosis or risk group. We will call them medical guideline states. Suppose that it is possible to establish relation between medical guidelines examination data, medical guideline actions and medical guideline states on the one side and input data of EHR on the other side. Suppose further that a translation table could express this relationship. EHR input data correspond to the medical guideline examination data or medical guideline actions. EHR input data, which correspond to the medical guideline states we call patient states.

What means, that some patient state or physician action at the input of EHR contradicts the medical guidelines? We suggest defining this contradiction in the following way. If some patient state or physician action at the input of EHR has its counterpart in medical guidelines and all occurrences of its counterpart in the medical guideline model are according to the content of EHR database unreachable, then this patient state or physician action contradicts medical guidelines.

\section{General Views on Comparing of Medical Guidelines with EHR}

EHR systems are now being developed in many ways in many places. EHR system enables to store all relevant data about patient in a structured way and facilitates their further computer processing [13]. The main part of each EHR are medical observations, laboratory results, stated diagnoses, medical treatments etc. The main purpose of EHR system is to store and retrieve data in userfriendly dialogue with physician. Advanced EHR systems should also utilize medical knowledge and provide following facilities.

Checking facility. We check if values of input data are in accordance with medical knowledge. Usually the system verifies if the input value is inside defined interval. 
Reminder facility. If a stated diagnosis or a chosen treatment is not in agreement with common medical knowledge, the system warns the user and suggests more probable diagnosis or more appropriate action.

Decision support facility. Based on EHR data the system supports decisionmaking activities of a physician.

Automatic checking of input data should be an integrated part of each electronic health record (EHR) system. EHR system should analyze input data and warn the user if he inputs data item which is out of interval of possible values or if he inputs data item, which suggests some decision or medical action, that contradicts common medical knowledge. For implementing this task, EHR system usually uses set of logical if - then rules. These rules could be extracted from text form of medical guidelines. The other possibility is to compare input data and medical guidelines directly using electronic medical guidelines. A method for direct comparing of EHR and medical guidelines was proposed in 12 .

We will concentrate on the reminder function of the EHR system. The usual way how to design reminder system is to design a set of rules, based on medical knowledge. These rules are then during user-EHR interaction checked against input data. The main problem is how to find out these rules. Usually they are to be built up by a team of experts and knowledge engineers. The methods of their elaboration can be similar to those used in building expert systems. When medical guidelines are at our disposal, the rules can be extracted from them. But is it necessary to follow this way? Perhaps it could be possible to compare data at the input of EHR system with medical guidelines directly.

EHR system can realize the reminder function itself or reminder function can be realized by a Stand-Alone Reminder System (SARS). The collaboration between EHR system and SARS could be described as follows:

- When user puts into EHR system a patient state or a physician action, EHR system sends an activation message to SARS. The part of the message must be the patient state or the physician action.

- SARS translates the patient state or the physical action into a medical guideline state or medical guideline activity according to the translation table.

- SARS, according to the guideline model, determines supports of all occurrences of medical guideline states or medical guidelines actions.

- SARS translates all EHR input data into medical guideline examinations according to the translation table.

SARS finds out if user follows electronic medical guideline. If not, it sends a warning message.

\section{Conclusion}

The advantages of proposed method of comparing medical guidelines and EHR using EFMG are the following.

General applicability. System based on proposed method can work with arbitrary guidelines. Only at first the guidelines must be transformed into GLIF 
graph model and then the model must be encoded into guideline describing language. The transformation of free text guidelines into GLIF model or some similar structured and precisely defined formal model should be accomplish anyway, because only in this way one can be certain, that guidelines are unambiguous and non-contradictory. The encoding of GLIF graph model into guideline describing language is then straightforward as each algorithm coding.

Facilitation of changes in the system. When some part of guidelines is changed, it is not necessary to correct the set of rules used for checking of input data. What is sufficient to do is making corresponding change in guideline model. Support of distributed computing. The EHR system can be running at one site and the system for comparing input data with guidelines at another site. The system based on the proposed method could be even more distributed. EFMG could be accessible for example on a server at department with medical experts, who worked out guidelines and are responsible for their maintenance and updating. The system for comparing EHR data and a medical guideline can be at another site and it could download EFMG from authorized site whenever necessary. In this case arises problem with translation table. Who should build and maintain it? The simplest solution could be sharing the same vocabulary. EHR system site could at the beginning inform comparing site which standard medical vocabulary, as SNOMED for example, is used. The first experience with comparing EHR data and EFMG on hypertension treatment has been gathered in both information system settings and in settings of the remote GP office.

\section{References}

1. Zvárová J., Hanzlíček P., Špidlen J.: Electronic Health Record in Cardiology: Pilot Application in the Czech Republic. In: MIST2002 Proceedings, Taiwan, John Wiley \& Sons (2002) 10-13

2. Iakovidis I.: Towards Personal Health Record: Current Situation, Obstacles and Trends in Implementation of Electronic Healthcare Record in Europe. Int. J. Med. Inform. 52 (1998) 105-115

3. Pierik F. H., Ginneken A. M., Timmers T., Stam H., Weber R. F.: Restructuring Routinely Collected Patient Data: ORCA Applied to Andrology. In: Bemmel J. H., McCray A. T. (eds.): Yearbook of Medical Informatics 98, Health Informatics and the Internet, Schattauer, Stuttgart (1998) 257-263

4. Přibík, V., Grünfeldová, H., Hanzlíček P., Peleška, J. and Zvárová J.:, Czech National Data Standards Implementations in ORCA Electronic Patient Record in Cardiology. In: Hasman A., Blobel B., Dudeck J., Engelbrecht R., Gell G., Prokosch H.U. (eds.) Medical Infobahn for Europe, IOS Press, Amsterdam (2000) 652-655

5. Svátek, V., Říha, A., Zíka, T., Zvárová J., Jiroušek, R., Zdráhal, Z.: Informal, Formal and Operational Modelling of Medical Guidelines. In: Proceedings $4^{\text {th }}$ JCKBSE , IOS Press, Amsterdam (2000) 87-92

6. Zvárová J., Peleška, J., Hanzlíček P. and Zvára, K.: Enhanced Care of Hypertensive Patients Using Internet. Technology and Health Care 6, Vol. 9, IOS Press (2001), $487-488$ 
7. Hanzlíček P.: Development of Universal Electronic Health Record in Cardiology. In: Surjan G., Engelbrecht R., McNair P. (eds.) Health Data in the Information Society, IOS Press, Amsterdam (2002) 356-360

8. Špidlen J., Hanzlíček P., Ř́íha, A., Zvárová J.: Flexible Information Storage in MUDR $^{\text {II }}$ EHR. Proceeding of the IJM EuroMISE 2004 (submitted)

9. Ohno-Machado L., Gennari, J. H., Murphy S.,N., Jain N.,L., Tu S., W., Oliver D., et al.: The GuideLine Interchange Format: A model for representing guidelines, Journal of the American Medical Informatics Association 5 (1998) 357-372

10. Boxwala A., A., Greenes R.,A., Debel S., R.: Architecture for Multipurpose Guideline Execution Engine, Proc. AMIA Symp. (1999) 701-705.

11. Peleg M., Boxwala A.,A., et al.: GLIF3: The Evolution of Guideline Representation Format, In: http:/smi-web.stanford.edu/projects/intermed-web/guidelines (2000)

12. Veselý A., Zvárová J., Peleška, J., Buchtela D., Anger Z.: Medical Guidelines Presentation and Comparing with Electronic Health Record. Proceeding of the IJM EuroMISE 2004 (submitted)

13. Ř́ha, A., Svátek, V., Němec P., Zvárová J.: Medical Guideline as Prior Knowledge in Electronic Healthcare Record Mining. In: Zanasi A., Brebbia C. A., Ebecken N. F. F. E., Melli P. (eds.): Data Mining III, WIT Press (2002) 809-818 